\title{
Anomalous variations of NDVI for a non-vegetated urban industrial area of Gumi, Korea
}

\author{
Kon Joon Bhang \\ Kumoh National Institute of Technology, Gumi, Korea
}

Email address:

bhang.1@kumoh.ac.kr

To cite this article:

Kon Joon Bhang. Anomalous Variations of NDVI for a Non-Vegetated Urban Industrial Area of Gumi, Korea. American Journal of Remote Sensing. Vol. 2, No. 6, 2014, pp. 44-49. doi: 10.11648/j.ajrs.20140206.11

\begin{abstract}
NDVI is often used to investigate how vegetation contributes to the surface urban heat islands (SUHI) effect. Generally, NDVIs obtained for partially vegetated or built-up areas have been found to be less than 0.4. However, anomalous NDVIs that are inconsistent with established concepts and relationships are frequently obtained for non-vegetated urban land cover. It is possible that these anomalous NDVIs have been distorted by the surface colors and patterns of rooftops. Therefore, we obtained NDVIs for an urban industrial area of Gumi, South Korea, and investigated how these NDVIs were affected by such surface colors and patterns. By assessing 148 factory rooftops, obtained NDVIs indeed showed a tendency to be affected by the surface colors and patterns of rooftops. A color classification of white, blue, purple, and red revealed that blue- and purple-colored rooftops resulted in higher NDVIs than other rooftops. Moreover, these rooftops were sometimes misidentified as forest and vegetation. The color tone, affected by brightness intensity, also contributed to the NDVIs obtained for blue- and purple-colored rooftops. Extreme cases showed that NDVIs obtained for rooftop surfaces could attain values indicative of dense vegetation (i.e., NDVIs $\geq 0.6$ ), when blue-colored rooftops were combined with complex surface patterns that generated more shadow. From these results, we concluded that the established relationship between LST and NDVI is likely to be invalid for non-vegetated urban industrial areas, and that NDVIs obtained for such areas should be used with caution, particularly in studies of the SUHI effect.
\end{abstract}

Keywords: Anomalous NDVI, Surface Temperature, Infrared Imaging, Urban, UHI, Surface, Landsat, Monitoring

\section{Introduction}

The normalized difference vegetation index (NDVI) has been widely used in many environmental monitoring applications such as surface urban heat island (SUHI) with satellite-derived images. Because SUHI is caused by the transformation from vegetation to other man-made structures, measuring the surface temperatures from urban areas has been one of the important issues in the UHI study. In UHI studies, NDVI has been frequently employed since it is very useful and informative in estimating land surface greenness by vegetation as well as simple to be calculated. Due these reasons, many environmental related issues took advantage of NDVI so that the NDVI-related studies provided us a critical insight on the importance of land cover change from vegetation to urban structures in many environmental issues.

For instance, the land surface temperature (LST) is a function of surface soil moisture content and vegetation cover [1]. Also, the amount of vegetation is strongly associated to
LST [2] [3]. Also, NDVI contributed to the LST calculations for the single-channel thermal infrared (TIR) imagery such as Landsat by providing surface material emissivity values to calculate LST. [4] found that thermal emissivity was logarithmically correlated with NDVI for natural surface and pointed out its potential use in energy balance with satellite thermal images. This was further studied by [5] that land surface emissivity was retrieved from NDVI to investigate the coupling of surface temperature and emissivity for soil and vegetation type covers. [6] and [7] developed a simplified approach to calculate surface temperature with surface emissivity derived from NDVI and analyzed the accuracy. The surface emissivity was measured using combination of NDVI, soil, and vegetation emissivity values. [8] attempted to retrieve energy balance estimations for agricultural regions by land surface emissivity driven from ASTER thermal images.

However, these studies with NDVI and surface temperature was problematic in urban areas since surface emissivity essential for calculating surface temperature primarily with 
soil and vegetation combination. Therefore, there are natural limitations due to the characteristics inherent to the simple combination of the red and near-infrared bands, especially in urban areas. For example, many studies showed that LST and NDVI are negatively correlated and this has been globally accepted in SUHI studies typically came with NDVI [9]. However, recent findings showed that the NDVI did not effectively portray the surface condition typically in urban areas [10]. Also, NDVI-LST correlation was dependent on both the season and the time of day rather than negatively associated. Strong negative correlations were found only in the warm seasons (i.e., during May-October), indicating that caution should be taken when relying on this well-known negative relationship during cold seasons [11].

In addition, we have often found that vegetation-like spectral properties of certain types of artificial land cover correspond to high NDVIs. In other words, NDVIs obtained for some types of urban cover are often greater than $0.4 \sim 0.5$, which is considered as the typical lower limit of pure vegetation. These anomalously high NDVIs have been used without notice or identification so that they may cause misinterpretation in the relationship between LST and vegetation or misidentification of land cover analyses for vegetation and urban land covers, typically in urban areas.

We attempted to reveal what causes these anomalous NDVIs and to understand how NDVIs and LSTs change with urban surface cover conditions. Specifically, we investigated how NDVIs, and their relationships with LSTs, were affected by surface colors and patterns triggered from [12]. One concern might be that NDVI is limited in its applicability to urban areas, because its primary purpose is to detect vegetation rather than to classify urban land cover. Furthermore, it may be necessary to obtain supplementary or secondary information to facilitate urban land cover analyses, because NDVI alone provides neither an adequate nor a complete measure of land cover properties within urban areas. Nonetheless, a sound understanding of basic NDVI properties is highly important because the NDVI is widely used, not only in analyses of vegetation but also urban land cover in environmental monitoring applications.

\section{Data Processing}

The study is focused on the largest industrial area packed with industrial facilities in Gumi, Korea. The center of the city is at $128^{\circ} 23^{\prime} 20.3^{\prime} \mathrm{N}, 36^{\circ} 06^{\prime} 20^{\prime} \mathrm{E}$ and the city is bisected by the Nakdong River from the north to the south. Datasets are two images of Landsat 5 TM obtained on June 15, 2009, and June 22, 2010, and a series of digital aerial photographs taken in October, 2011.

As shown in Figure 1, data processing can be divided into three image processing categories; (1) atmospheric correction, (2) primary component extraction, (3) NDVI and brightness temperature extraction, (4) and rooftop color and pattern identification.

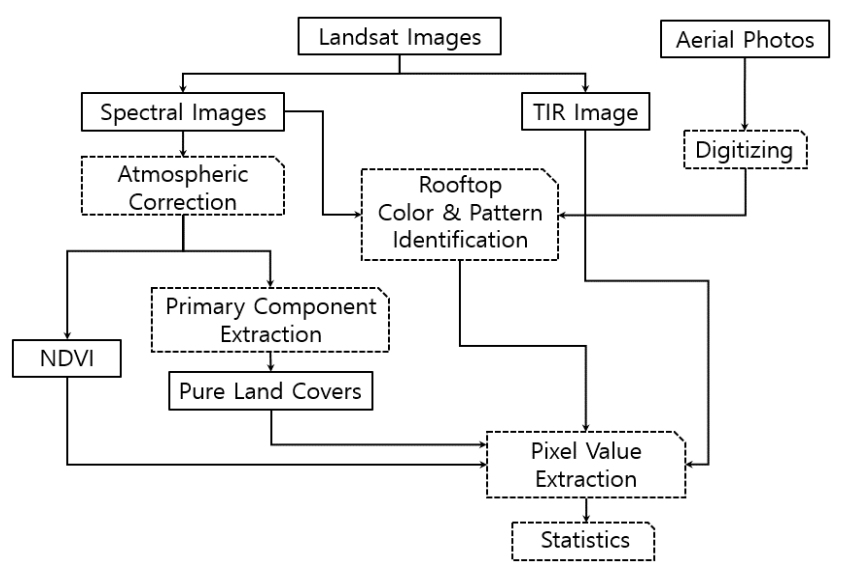

Figure 1. Data processing

\subsection{Atmospheric Correction and Classification}

We followed the typical atmospheric correction method using the FLAASH module in the ENVI software package for band 1 through 5 and 7 of the two Landsat images. The input parameters of the atmospheric conditions for humidity, cloud, visibility, etc. were retrieved from the meteorological database from Korea Meteorological Administration based on mid-latitude summer model with water absorption feature of $1,135 \mathrm{~nm}$ and 2-band (K-T) aerosol retrieval with the actual approximate visibility of $40 \mathrm{~km}$.

\subsection{Primary Component Extraction}

The pure land covers of soil, vegetation, and water were then segmented using a scatterplot with band 3 and 4 [13]. In Figure 2, pure soil pixels (green dots) were packed at the lowest part of the plot in a small area but pure vegetation (red dots) was rather distributed along the vertical axis of band 4 . Water was located at a highly concentrated area of the diagonal end near the horizontal axis.

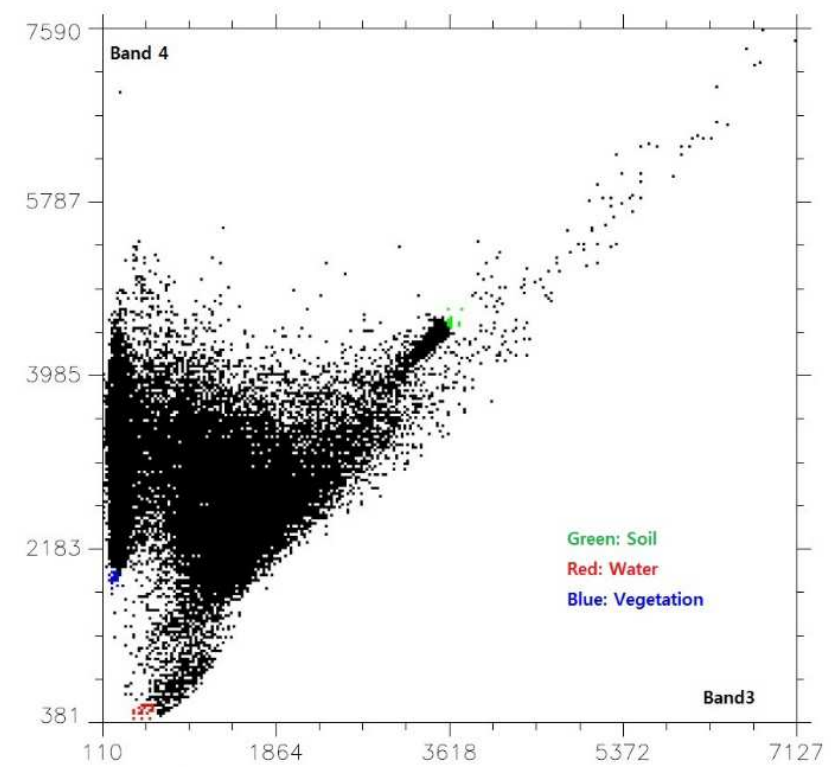

Figure 2. Primary component extraction for soil, water, and vegetation. 


\subsection{NDVI and Brightness Temperature}

The atmospherically corrected Landsat images of the red and near-IR bands were used to calculate NDVI with the following equation.

$$
\text { NDVI }=\frac{\rho_{4}-\rho_{3}}{\rho_{4}+\rho_{3}}
$$

where $\rho_{3}$ and $\rho_{4}$ are reflectance of TM band 3 and 4 , respectively.

The calculation of LST with the TIR band of Landsat 5 TM requires surface emissivity which must be determined before the LST calculation. We used brightness temperature instead to simplify the processing as described in [14]. The brightness temperature (or the effective at-satellite temperature) in Kelvin can be expressed as the following.

$$
B(T)=\frac{K_{2}}{\ln \left(\frac{K_{1}}{L}+1\right)}
$$

where $K_{1}=607.76$ and $K_{2}=1260.56$ for Landsat $5 \mathrm{TM}$, and $L$ is the spectral radiance.

\subsection{Rooftop Color and Pattern Identification}

The most important data processing is to separate urban land covers by their specific characteristics. Unlike soil, water, and vegetation, man-made materials were complex due to the mixture of various materials in each individual pixel and they hindered characterizing the condition of each pure material. Therefore, we selected most frequently appearing covers in the study area such as large building rooftops and asphalt enough to be covered by Landsat image spatial resolution of $30 \mathrm{~m}$.

Aerial photographs with $50 \mathrm{~cm}$ spatial resolution were manually digitized for the Landsat image pixel extraction of asphalt and buildings with areas greater than $3,600 \mathrm{~m}^{2}$, such that they included at least one rooftop pixel in Landsat images with $30 \mathrm{~m}$ resolution. The 148 digitized rooftops in total were then identified by the surface color and pattern.

The Landsat rooftop pixels were then divided into subsets of gray, blue, green, and red color families based on designed color groups in which the digitally assigned pixel values (DNs) of Landsat bands 3, 2, and 1 were divided into 8 classes for each RGB color band, respectively. Since the Landsat images (the 8 bit TIFF image format) carry 256 levels of radiometric intensity for each band, total 32 classes for each band produced total 512 color groups by mixing the RGB values for the rooftop pixels. However, the rooftops in the study area could be typically characterized by blue, purple, and red colors with various brightness intensity. Therefore, only the three color groups with 6 levels of brightness intensity were taken into account as identified rooftop colors. Also, the digitized rooftops were categorized by the number of ridges, allowing for identification of rooftop patterns. The land covers of soil, water, vegetation, asphalt and rooftop were finally used to extract pixel values of NDVI and LST for each land cover.

\section{Results}

\subsection{NDVI Characteristics in Urban Areas}

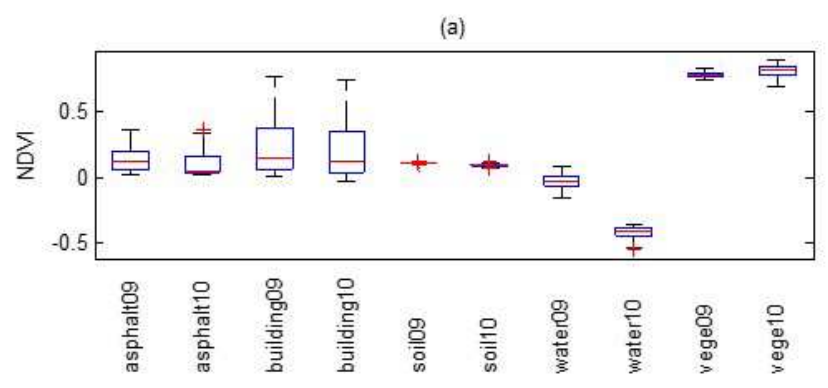

(b)

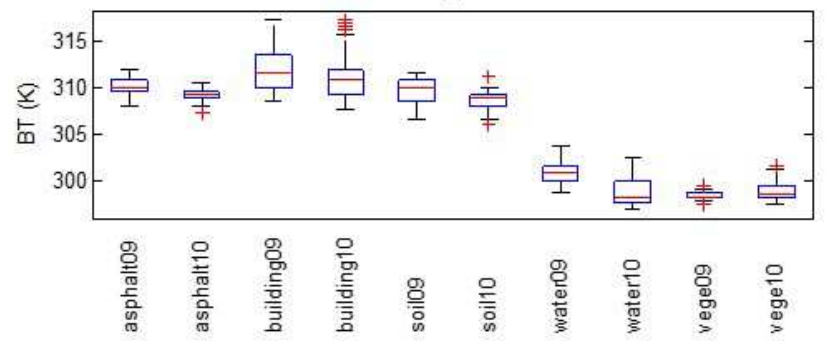

Figure 3. Box plots indicating medians and quartiles for (a) NDVI and (b) brightness temperature for the land cover types asphalt, rooftop, soil, water, and vegetation from June 15, 2009 and June 22, 2010.

The distributions and averages of NDVIs and LSTs for the five land cover categories in the present study are presented as box plots in Figure 3. The average NDVIs obtained for forest vegetation on the Landsat 5 TM images obtained on June 15, 2009 , and June 22, 2010, were 0.78 and 0.81 with very narrow distributions, respectively (Table 1 and Figure 3). Because of these high NDVIs (Figure 3a), forest vegetation could be more easily differentiated than the other land cover types. Obtained NDVIs were below zero for water, even though the averages for the two images were very different. This is because the Nakdong River running through the study area was under rehabilitation from early 2009 to increase its width and depth, which made the corresponding pixels darker in the 2010 image. The NDVIs obtained for vegetation and water were positive and negative, respectively, for both images although LSTs found for these covers were in a similar range (Figure $3 b)$. The average NDVI values obtained for both soil and rooftops were 0.11 and 0.26 for 2009 images, respectively and 0.1 and 0.22 for 2010 images, respectively. The soil in the study area was homogeneous, causing a tightly packed NDVI distribution. However, building rooftops had various surface properties, resulting in a wide NDVI distribution. LSTs were higher for urban covers than for natural covers, such that LST was highest for rooftops, followed by asphalt, and lowest for soil.

\subsection{High NDVIs for Rooftops and Asphalt}

Many NDVIs obtained for the rooftop pixels in the Landsat 
5 TM images from June 15, 2009, and June 22, 2010, appeared to be indicative of vegetation (i.e., NDVI $>0.5$ ). The basic mechanism of NDVI is based on the spectral capability of the near-infrared channel detecting chlorophyll in vegetation. In this respect, high NDVI values for the natural land covers such as soil, water, and vegetation, should include NIR-responsive materials, in natural, typically vegetation. Therefore, many UHI studies have claimed that LST has a negative relationship with existing vegetation cover, which was assumed to be correctly reflected by obtained NDVIs. Although this is certainly true for natural land covers with soil and vegetation, it may not be for urban areas. Figure 4, which shows NDVIs and LSTs with aerial photographs that covered part of the study area, illustrates this issue. The rooftop in the red circle at the upper left corner in Figure 4a demonstrates high NDVIs, i.e., 0.75 and 0.71 for June 15, 2009, and June 22, 2010, respectively. Various NDVIs were obtained for other blue rooftops, such as the rectangular boxes showing NDVIs of $0.32,0.15,0.56$, and 0.4 (Figure 4a). Rooftops exhibiting high NDVIs had relatively high LSTs (i.e., in the range 308.5-310.3 K; average of $303.1 \mathrm{~K}$ ); however, rooftops exhibiting low NDVIs also had relatively high LSTs. In other words, LSTs were high regardless of NVDI values, implying that the NDVI-LST relationship did not exhibit the typical negative correlation between NDVI (i.e., the amount of vegetation) and LST.

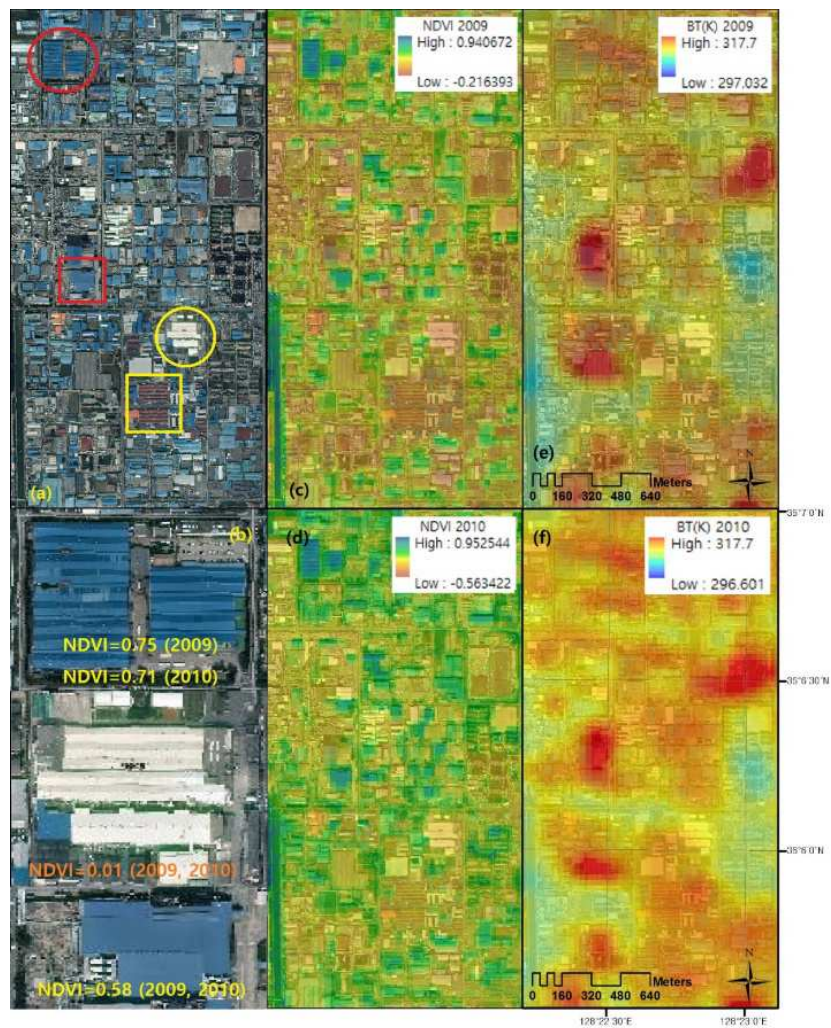

Figure 4. Factory rooftops illustrating the various surface pattern in the study area. (a) circles and squares denote rooftop samples for which high and low NDVIs were obtained, respectively; (b) rooftop samples in the upper left circle for the maximum and minimum NDVIs; (c), (d) NDVI maps; and (e), (f) temperature maps for 2009 and 2010 Landsat 5 TM image.
Through inspection of blue rooftops, we were able to identify certain NDVI trends over the NDVI range obtained for blue rooftops (i.e., from 0.0 to approximately 0.8 ). Identifiable causes of these trends were the color and color tone of rooftops and the geometric patterns of rooftop surfaces (Figure $4 a-4 b)$.
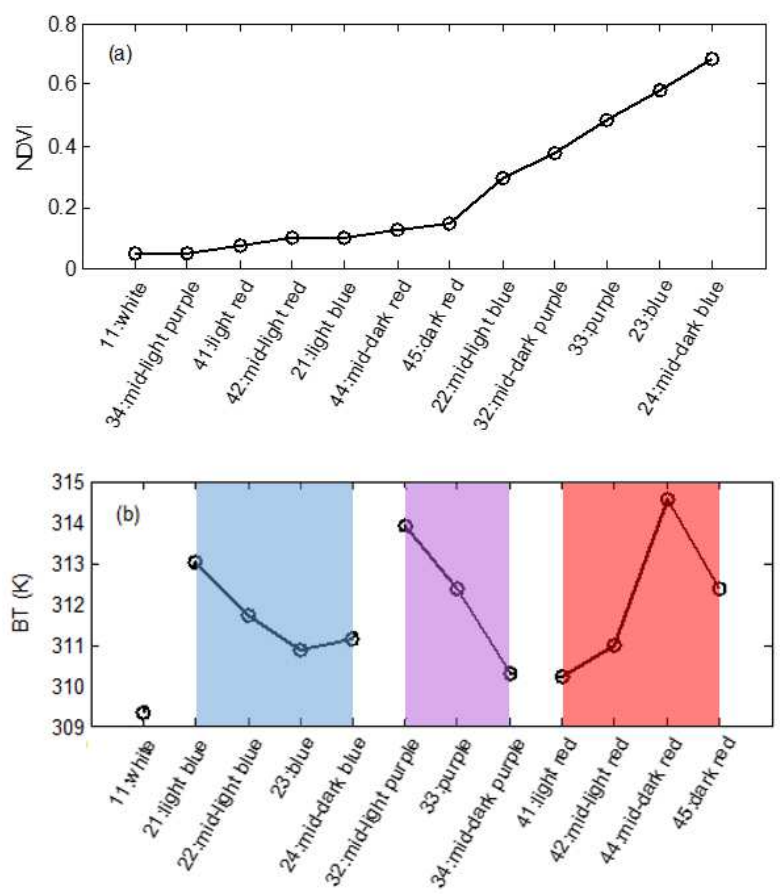

Figure 5. Relationships between color and (a) NDVI and (b) brightness temperature of the urban surface. Numbers in front of each color name on the $x$-axis indicate color code for the first digit and color intensity for the second digit encoded for classification.

To quantify these influences for NDVIs, rooftop pixel coloring was encoded with the four colors found most frequently within the study area based on the color identification as described in the methodology: 1 corresponds to grey or white, 2 corresponds to blue, 3 corresponds to purple, and 4 corresponds to red. Furthermore, the tone (or brightness intensity) was also encoded as follows: 1 corresponds to light, 2 corresponds to mid-light, 3 corresponds to original, 4 corresponds to mid-dark, and 5 corresponds to dark. This scheme of encoding was based on the digital color table using RGB values, by segmenting 256 values into five groups for the RGB bands (i.e., bands 3, 2, and 1, respectively). The fact that rooftop surface shapes were also characterized by the number of ridges for each of the 148 digitized rooftops was also taken into account by encoding them such that $0,1,2$, and 3 corresponded to a flat rooftop, a rooftop with one ridge, a rooftop with two ridges, and a rooftop with three or more ridges, respectively.

Figure 5a shows that an increasing trend of NDVI was found in relation to colors and color tone of rooftops, illustrating that NDVIs obtained for grey and red rooftops were typically low compared to those for purple and blue rooftops. The highest NDVIs occurred for blue rooftops, followed by purple, red, and grey rooftops. Furthermore, it 
was found that strong brightness intensity and light color tone were commensurate with low NDVIs, except in the case of red rooftops (Figure $5 \mathrm{~b}$ ). In other words, the temperature appeared to have trends depending on colors and color tones so that blue and purple rooftops exhibited decreasing trends as color tone decreased, whereas LSTs tended to increase with decreasing color tone for the red rooftops (Figure 5b).

Table 1. Statistical values for NDVI and Temperature. Average NDVI especially for forest vegetation is distinguishable with 0.78 and 0.81 .

\begin{tabular}{llllllllllll}
\hline & Vegetation & \multicolumn{1}{l}{ Water } & Soil & \multicolumn{4}{c}{ Asphalt } & \multicolumn{2}{c}{ Rooftop } \\
\hline & 2009 & 2010 & 2009 & 2010 & 2009 & 2010 & 2009 & 2010 & 2009 & 2010 \\
Mean & 0.78 & 0.81 & -0.03 & -0.44 & 0.11 & 0.10 & 0.14 & 0.10 & 0.26 & 0.22 \\
RMSE & 0.02 & 0.04 & 0.06 & 0.05 & 0.01 & 0.01 & 0.09 & 0.09 & 0.23 & 0.22 \\
& Temperature $(\mathrm{K})$ & & & & & & & \\
Mean & 298.4 & 298.8 & 310.0 & 298.8 & 309.7 & 308.5 & 310.1 & 309.3 & 312.1 & 310.9 \\
RMSE & 0.4 & 0.8 & 1.3 & 1.4 & 1.4 & 1.2 & 0.9 & 0.7 & 2.1 & 2.2 \\
\hline
\end{tabular}
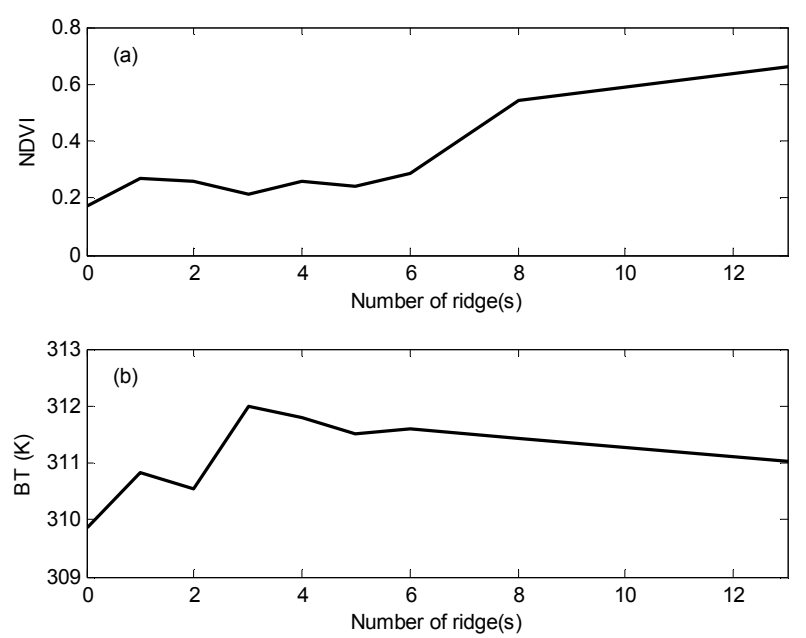

Figure 6. Relationships between (a) NDVI and number of ridges and (b) surface brightness temperature and number of ridges

Figure 6 illustrates the effect of surface patterns on NDVIs and LSTs for blue rooftops. The NDVIs were found to increase as the number of ridges increases, although fluctuations in LSTs did not seem to affect rooftop surface patterns. It is apparent that the complex washboard rooftop pattern suggests a greater risk of colors being affected by shadow, resulting in darker coloring overall compared to a simple or flat rooftop pattern. This may cause higher NDVIs and lower LSTs for some cases, although there may be no association between the NDVIs and LSTs themselves.

\section{Conclusion}

NDVIs have often been used to investigate how vegetation contributes to the SUHI effect. Generally, NDVIs obtained for partially vegetated or built-up areas have been found to be less than 0.4. However, anomalous NDVIs that are inconsistent with established concepts and relationships are frequently obtained for non-vegetated urban land cover. Because it is possible that these anomalous NDVIs were distorted by the surface colors and patterns of rooftops, we investigated how these NDVIs were affected by these factors. By assessing 148 factory rooftops, each with a surface area greater than 3,600 $\mathrm{m}^{2}$, it was revealed that obtained NDVIs indeed showed a tendency to be affected by surface colors and patterns of rooftops. A color classification of white, blue, purple, and red revealed that blue- and purple-colored rooftops resulted in higher NDVIs compared to other rooftops. Moreover, these rooftops were sometimes misidentified as forest vegetation. The color tone, which is affected by brightness intensity, also contributed to the NDVIs obtained for the blue- and purple-colored rooftops. Extreme cases showed that NDVIs obtained for rooftop surfaces could attain values indicative of dense vegetation (i.e., NDVI $\geq 0.6$ ), when blue-colored rooftops were combined with complex surface patterns that generated more shadow. LST and NDVI did not exhibit negative correlation, deviating from the well-established negative relationship on which the majority of monitoring applications relies. We concluded that the established relationship between LST and NDVI is likely invalid for non-vegetated urban industrial areas, and that NDVIs obtained for such areas should be used with caution, particularly in studies of the SUHI effect.

\section{Acknowledgement}

This paper was supported by Research Fund, Kumoh National Institute of Technology.

\section{References}

[1] T.W. Owen, T.N. Carlson, and R.R. Gillies. An assessment of satellite remotely-sensed land cover parameters in quantitatively describing the climatic effect of urbanization. Int J. Remote Sens., vol. 19, no. 9, pp. 1663-1681, Jan. 1998.

[2] C.P. Lo, D.A. Quattrochi, and J.C. Luvall. Application of high resolution thermal infrared remote sensing and GIS to assess the urban heat island effect. Int. J. Remote Sens., vol. 19, no. 2, pp. 287-304, Feb. 1997.

[3] J.S. Wilson, M. Glay, E. Martin, D. Stuckey, and K. Vedder-Risch. Evaluating environmental influences of zoning in urban ecosystems with remote sensing. Remote Sens. Environ., 86, no. 3, pp. 303-321, Aug. 2003.

[4] A.A. Van De Griend and M. Owe. On the relationship between thermal emissivity and the normalized difference vegetation index for natural surfaces. Int. J. Remote Sens., vol. 14, no. 6, pp. 1119-1131, May 1993.

[5] E. Valor and V. Caselles., Mapping land surface emissivity from NDVI: Application to European, African, and South American areas. Remote Sens. Environ., vol. 57, no. 3, pp. 167-184, Sep. 1996.

[6] J.A. Sobrino, N. Raissouni, and Z.L, Li. A comparative study of land surface emissivity retrieval from NOAA data. Remote Sens. Environ., vol. 75, no. 75, pp. 256-266, Feb. 2001.

[7] J.A. Sobrino, J.C. Jiménez-Muñoz, G. Soria, M. Romaguera, L. Guanter, J. Moreno, A. Plaza, and P. Martinez. Land surface emissivity retrieval from different VNIR and TIR sensors. IEEE Trans. Geosci. Remote Sens., vol. 46, no. 2, pp. 316-327, Feb. 2008. 
[8] J.C. Jiménez-Muñoz, J.A. Sobrino, A. Gillespie, D. Sabol, and W.T. Gustafson. Improved land surface emissivities over agricultural areas using ASTER NDVI, Remote Sens. Environ., vol. 103, no. 4, pp. 474-487, Aug. 2006.

[9] K.P. Gallo, and J.D. Tarpley. The comparison of vegetation index and surface temperature composites for urban heat-island analysis. Int. J. Remote Sens., vol. 17, no. 15, pp. 3071-3076, Oct. 1996.

[10] F. Yuan and M.E. Bauer. Comparison of impervious surface area and normalized difference vegetation index as indicators of surface urban heat island effects in Landsat imagery. Remote Sens. Environ., vol. 106, no. 3, pp. 375-376, Feb. 2007.

[11] D. Sun and M. Kafatos. Note on the NDVI-LST relationship and the use of temperature-related drought indices over North
America. Geophys. Res. Lett., vol. 34, no. 24, pp. L24406-L24500, Jan. 2007.

[12] K.J. Bhang and S.S. Park. Evaluation of the surface temperature variation with surface settings on the urban heat island in Seoul, Korea, using Landsat-7 and SPOT. IEEE Geosci. Remote Sens. Lett., vol. 6, no. 4, pp. 708-712, Oct. 2009.

[13] D.A. Roberts, M. Gardner, R. Church, S. Ustin, G. Scheer, and R.O. Green, R.O. Mapping chaparral in the Santa Monica Mountains using multiple endmember spectral mixture models. Remote Sens. Environ., vol. 65, no. 3, pp. 267-279, Sep. 1998.

[14] NASA. Chapter 11 3.3. Band 6 Conversion to Temperature, Landsat 7 Science Data Users Handbook, 2011. 